\title{
Superfluid Interfaces in Quantum Solids
}

\author{
Evgeni Burovski, ${ }^{1}$ Evgeni Kozik, ${ }^{1}$ Anatoly Kuklov, ${ }^{2}$ Nikolay Prokof'ev, ${ }^{1,3}$ and Boris Svistunov ${ }^{1,3}$ \\ ${ }^{1}$ Department of Physics, University of Massachusetts, Amherst, Massachusetts 01003, USA \\ ${ }^{2}$ Department of Engineering Science and Physics, The College of Staten Island, City University of New York, \\ Staten Island, New York 10314, USA \\ ${ }^{3}$ Russian Research Center, "Kurchatov Institute," 123182 Moscow, Russia
}

(Received 22 December 2004; published 28 April 2005)

\begin{abstract}
One scenario for the nonclassical moment of inertia of solid ${ }^{4} \mathrm{He}$ discovered by Kim and Chan [Nature (London) 427, 225 (2004)] is the superfluidity of microcrystallite interfaces. On the basis of the most simple model of a quantum crystal - the checkerboard lattice solid - we show that the superfluidity of interfaces between solid domains can exist in a wide range of parameters. At strong enough interparticle interaction, a superfluid interface becomes an insulator via a quantum phase transition. Under the conditions of particle-hole symmetry, the transition is of the standard $U(1)$ universality class in $3 \mathrm{D}$, while in $2 \mathrm{D}$ the onset of superfluidity is accompanied by the interface roughening, driven by fractionally charged topological excitations.
\end{abstract}

PACS numbers: 67.40.-w, 05.30.-d, 67.80.-s

Recent observation by Kim and Chan [1] of the nonclassical moment of inertia (NCMI) of ${ }^{4} \mathrm{He}$ at pressures significantly higher than the solidification point is a breathtaking result, especially striking in view of the theoremlike theoretical arguments against the existence of commensurate supersolids [2]. The fact of commensurabilityequivalently, one may put it as the absence of vacancies, interstitials, or both - of the equilibrium solid ${ }^{4} \mathrm{He}$ at $T=$ 0 is supported by extensive experimental work over the past several decades (for review, see, e.g., [3]), as well as by the most recent experimental and numeric studies [4,5]. The commensurability of solid ${ }^{4} \mathrm{He}$ rules out NCMI based on Bose-Einstein condensation of vacancies [6]. Two of us have proposed recently [2] that NCMI might be due to the superfluidity of interfaces between ${ }^{4} \mathrm{He}$ crystallites. At present, the weak point of this hypothesis is the absence of a theoretical analysis and/or direct experimental evidence of the superfluidity in the walls separating insulating domains.

The problem of interface superfluidity in a quantum solid is of significant general interest in its own right, being potentially relevant not only to the solid ${ }^{4} \mathrm{He}$ polycrystal, but also to the properties of domain walls in spin arrays and ultracold atoms in optical lattices.

In this Letter, we present a proof-of-principle study of superfluidity in interfaces between insulating domains with broken translation symmetry (solids). We address the problem by studying the checkerboard lattice solid (CB). We start with giving a simple illustrative theoretical argument that at least under certain limiting conditions the domain wall in our system has to be superfluid. Our numeric simulations of 2D and 3D models reveal superfluidity of the $\mathrm{CB}$ domain walls in a large range of parameters. We pay special attention to the study of the superfluid (SF)insulator (I) quantum phase transition in the interface. In $3 \mathrm{D}$, the transition turns out to be in the $U(1)$ universality class. In 2D, we conclude that the I-to-SF transition in the wall is driven by proliferation of topological excitations that carry the fractional particle charge $1 / 2$, as well as the quantum of the interface shift in the transversal direction. The latter circumstance results in an interesting effect: The transition is accompanied by the wall roughening.

The simplest system featuring both superfluid and CB phases is that of the hard-core lattice bosons with nearestneighbor repulsion, at half-integer filling factor (see, e.g., [7], and references therein). The model can be exactly mapped onto spin-1/2 XXZ antiferromagnet, which leads to the following correspondence. The $\mathrm{CB}$ phase is equivalent to the easy-axis antiferromagnet (characterized by the broken $Z_{2}$ symmetry), while the SF phase is identified with the easy-plane antiferromagnet (characterized by broken $U(1)$ symmetry). Correspondingly, in terms of the Néel vector, $\vec{S}$, the CB order parameter (i.e., staggered magnetization) is $M=S_{z}$, while the SF order parameter is $\Psi=$ $S_{x}+i S_{y}$. Generically, the ground state of the model is either SF or CB, depending on the Hamiltonian parameters (we work at half filling only, and the $\mathrm{CB}+\mathrm{SF}$ phase separation issue [8] is thus irrelevant). There is also a special $S U(2)$-symmetric Heisenberg point. In $2 \mathrm{D}$ and 3D ground states of the Heisenberg Hamiltonian the $S U(2)$ symmetry is broken, so that the vector $\vec{S}$ is nonzero and can point at any direction.

Let us take now, say, a 3D system with an easy-axis Hamiltonian that is very close to the Heisenberg point $(T=0)$, and create two large domains, $S_{z}=M$ and $S_{z}=$ $-M$. What is the structure of the domain wall? Being close to the Heisenberg point, we are forced to conclude that the wall is very thick in the transverse direction, with the vector $\vec{S}$ well defined locally inside the wall and evolving smoothly from $(0,0, M)$ to $(0,0,-M)$ across the wall. The energetic cost is controlled by the closeness to the Heisenberg point and can be rendered arbitrarily small. In the middle of the wall, $\vec{S}=\left(S_{x}, S_{y}, 0\right)$, which means that 
the wall is characterized by broken $U(1)$ symmetry implying superfluidity in the bosonic case. As the system is taken deeper into the solid state in the bulk, it becomes energetically favorable to suppress the module of $\vec{S}$ to zero in the wall, which means an insulating state of the wall. Similar transformation of the domain wall-from the Bloch-type, where $|\vec{S}|$ is finite everywhere, to the Ising-type, where $|\vec{S}|=0$ is in the middle of the wall-takes place in classical ferromagnets close to the Curie point [9].

Once the superfluidity of a domain wall is established in the limiting case, one may expect that it can take place under more general conditions, especially in view of the following, almost obvious, energetic argument: Because of geometrical frustration, the energy cost to translate a particle along the wall is less than in the bulk. For example, in the case of the 2D CB solid, the particle jump increases the energy by $\sim 3 V$, where $V>0$ is the nearest neighbor interaction energy. A jump of a particle at the wall [see, e.g., Fig. 4(a)] costs only $\sim V$.

A point of concern, however, is that generically the transition from SF to CB is of the first order so that for a given system or range of parameters it may turn out that the interface between two insulating domains is always in the insulating state.

To get an idea of how likely it is to get the interface between the two CB domains superfluid, we simulate a domain wall in the bond-current model - a discrete-imaginary-time analog of a $(d+1)$-dimensional world line representation of a quantum bosonic or spin system in $d$ spatial dimensions [10]. The Hamiltonian of the model reads

$$
H=t \sum_{\mathbf{n}} \sum_{\alpha=1}^{d} J_{\mathbf{n}, \alpha}^{2}+2 p \sum_{\langle\mathbf{n}, \mathbf{m}\rangle}\left(J_{\mathbf{n}, \tau}-\frac{1}{2}\right)\left(J_{\mathbf{m}, \tau}-\frac{1}{2}\right) .
$$

Here the integer vector $\mathbf{n}=\left(n_{1}, \ldots, n_{d}, n_{\tau}\right)$ labels sites of the $(d+1)$-dimensional cubic lattice, $\alpha=1, \ldots, d$ enumerates the spatial directions, and $\tau$ denotes the temporal direction. The dimensionless parameters $t$ and $p$ are related to the hopping amplitude and the nearest-neighbor interaction of the corresponding quantum model, respectively [10]. Currents $J_{\mathbf{n}, \alpha}$ and $J_{\mathbf{n}, \tau}$ are integers associated with bonds adjacent to the site $\mathbf{n}$ in directions $\alpha$ and $\tau$, respectively. The summation in the second term runs over all pairs of temporal bonds having a common plaquette. The configurations of bond currents are subject to the zerodivergence constraint. Without loss of universality, we restrict the values of bond currents to $J_{\mathbf{n}, \alpha}=0, \pm 1$, and $J_{\mathbf{n}, \tau}=0,1$. The zero-divergence constraint then reads

$$
\sum_{\alpha}\left(J_{\mathbf{n}, \alpha}+J_{\mathbf{n},-\alpha}\right)+\left(J_{\mathbf{n}, \tau}+J_{\mathbf{n},-\tau}\right)=0,
$$

where the negative sign means the opposite direction, so that $J_{\mathbf{n},-\alpha} \equiv-J_{(\mathbf{n}-\hat{\alpha}), \alpha}$ and $J_{\mathbf{n},-\tau} \equiv-J_{(\mathbf{n}-\hat{\tau}), \tau}$; the hats stand for unit vectors in the corresponding directions.
We simulate the model (1), using the Worm algorithm [11], in the range of parameters where the bulk is deep into the solid regime. To automatically create a domain wall, we take a lattice with periodic boundary conditions and an odd number of sites in the spatial direction $x$ (system sizes in all the other directions are even). The domain wall superfluidity manifests itself as a nonzero mean square of winding numbers [12] in the direction(s) parallel to the wall: $\left\langle W_{\|}^{2}\right\rangle \neq 0$, while $\left\langle W_{x}^{2}\right\rangle=0$. More specifically, for a $3 \mathrm{D}$ system with the domain wall in the $y z$ plane, the superfluid stiffness is given by $\rho_{s}=\left\langle W_{y}^{2}+W_{z}^{2}\right\rangle / 2 L_{\tau}$, and the compressibility is likewise defined in terms of the winding numbers in temporal direction [12]. In a 2D system with the domain wall in the $y$ direction the statistics of winding numbers is essentially discrete, $P\left(W_{y}\right) \propto$ $\exp \left[-\left(L_{y} / L_{\tau}\right) W_{y}^{2} / 2 \rho_{s}\right]$, and an appropriate estimator for the superfluid stiffness is $\rho_{s}^{-1}=-2\left(L_{\tau} / L_{y}\right) \times$ $\ln \left[P\left(W_{y}=1\right) / P\left(W_{y}=0\right)\right]$, and likewise for the compressibility. Here $L_{\tau}$ and $L_{y}$ are the linear system sizes in the corresponding directions. In our simulations, we set $L_{y}=L_{z}=L_{\tau}=L$ and $L_{x}=L+1$.

In 3D, we found that for $t \leqslant 1$ the interfaces are never superfluid. Simulations at $t=1.3$ revealed a first-order SFCB transition in the bulk at $p \approx 0.2$ with the interface remaining superfluid until $p$ becomes equal to $p_{c}=$ $0.2711(5)$. In $2 \mathrm{D}$, the simulations were performed at $t=$ 0.9. The first-order bulk SF-CB transition was found at $p \approx 0.5$, while the interface becomes insulating only at $p_{c}=0.7667(5)$.

A superfluid interface embedded into a $d$-dimensional solid is a $(d-1)$-dimensional superfluid, and the scenario for the quantum phase transition from SF to I state in such a system is interesting on its own. Our model, Eq. (1), has a particle-hole symmetry. Thus, commensurability should play a key part in the criticality [13]. Fundamentally, there are two qualitatively different cases, depending on whether the interface is smooth or rough. If the interface is centered at $x=0$ and $x(\vec{\rho})$ is its instantaneous shape ( $\vec{\rho}$ is the vector in the hyperplane perpendicular to the $x$ axis), then, by definition, $\left\langle x(\vec{\rho})^{2}\right\rangle \sim 1$ for a smooth interface, while for a rough interface $\left\langle x(\vec{\rho})^{2}\right\rangle$ is macroscopically large (scales as some power of the system size). For a smooth interface, the $\mathrm{CB}$ environment plays a role of a periodic external potential that doubles the interface unit cell. This means that a smooth interface can be treated as a commensurate system with an integer (unity) filling factor. Its SF-I transition then corresponds to the superfluid-Mott insulator (MI) transition at integer filling, known to be of the $U(1)$ universality class [14]. If the interface is rough, then the effect of the solid environment is averaged out by the zero-point fluctuations of $x(\vec{\rho})$ and the effective filling factor for the interface remains half-integer, with corresponding implications for the universality class of the SF-I quantum phase transition.

In our simulations, we observe the smooth-interface scenario in $3 \mathrm{D}$ and the rough-interface scenario in $2 \mathrm{D}$. 


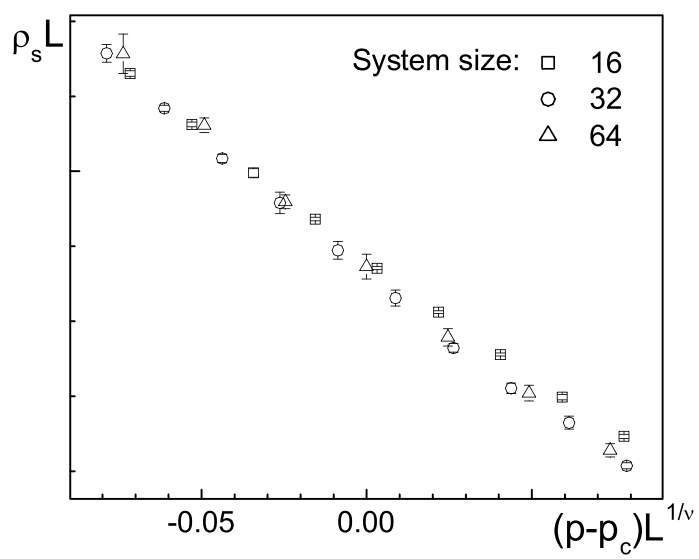

FIG. 1. Criticality for the interface in a 3D system. The data for $\rho_{s}$ as a function of the parameter $p$ is consistent with the exponent $\nu \approx 0.671$ of the $U(1)$ universality class.

In Fig. 1 we present the 3D data in the vicinity of the critical point, $p_{c}=0.2711(5)$. A very good data collapse with the critical exponent $\nu \approx 0.671$ of the $U(1)$ universality class is indicative of the standard SF-MI scenario. In 2D, the interface forms a 1D Luttinger liquid, the main characteristic of which is the dimensionless parameter $g=$ $\pi \sqrt{\rho_{s} \kappa}$, where $\rho_{s}$ and $\kappa$ are the 1D superfluid stiffness and compressibility, respectively. In a Luttinger liquid with a filling factor $1 / m$ ( $m$ is an integer) the SF-I (KosterlitzThouless type) transition takes place at $g=g_{c}=2 / \mathrm{m}^{2}$ [15]. As we argued above, a smooth interface implies $m=$ 1 and, correspondingly, criticality at $g_{c}=2$. The results of our simulation show that the SF-I transition actually takes place at $g_{c}=1 / 2$, corresponding to the half-filling case, implying the rough-interface scenario.

As is always the case with SF-I transitions in 1D systems, a brute-force numeric observation of the critical $g_{c}$ is problematic in view of the exponentially divergent correlation length. We thus need to perform the finite-size analysis of the data using Kosterlitz-Thouless renormalization-group flow:

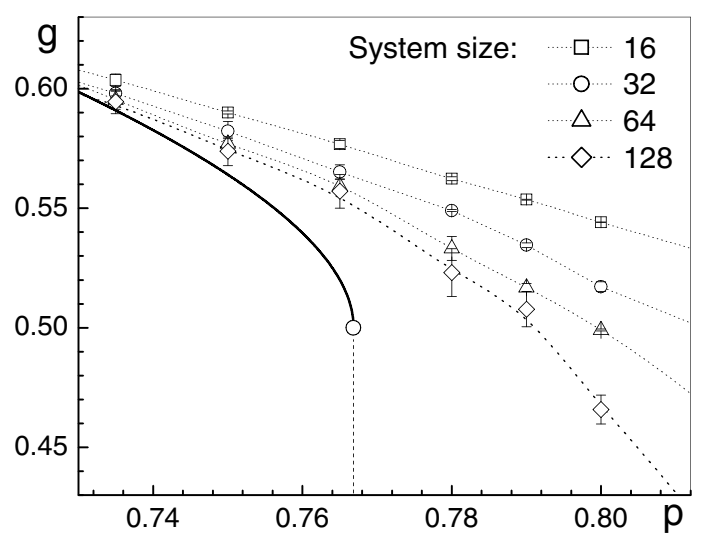

FIG. 2. Luttinger-liquid parameter $g$ as a function of $p$. Dotted lines are to guide the eye. The solid line is the KosterlitzThouless extrapolation to the infinite system size.

$$
\int_{g\left(L_{1}\right) / g_{c}}^{g\left(L_{2}\right) / g_{c}} \frac{d t}{t^{2}(\ln t-\xi)+t}=4 \ln \left(L_{1} / L_{2}\right)
$$

Here $g(L)$ is the Luttinger parameter $g$ as a function of the system size, $\xi$ is an $L$-independent microscopic parameter (which is an analytic function of $p$ ). At a given $p$, the value of $\xi$ is obtained with Eq. (3) from numeric values of $g\left(L_{1}\right)$ and $g\left(L_{2}\right)$. The consistency with the Kosterlitz-Thouless renormalization-group flow is checked by the data collapse for different pairs of system sizes and also by the shape of the curve $\xi(p)$, which should look as a straight line in the vicinity of the critical point, in contrast to the $g(L, p)$ curves - as functions of $p$, at large enough $L$. These curves should demonstrate a considerable curvature consistent with a (slow) evolution, as $L \rightarrow \infty$, towards the jump at $p_{c}$ from $g=1 / 2$ to $g=0$. These features are seen in Figs. 2 and 3. After extracting the function $\xi(p)$, the macroscopic $g(p) \equiv g(L \rightarrow \infty, p)$ limit is obtained-in accordance with Eq. (3)-from $\left(g / g_{c}\right)\left(\ln g / g_{c}-\xi\right)=$ -1 . The critical value $g=1 / 2$ implies a rough interface, since roughening is apparently the only mechanism of eliminating the effect of broken translation symmetry in the bulk. There is also a strong argument in favor of the simultaneous appearance of superfluidity and roughening in the 1D interface. There is little doubt that deep in the insulating phase the interface becomes smooth $(T=0)$, since roughening costs finite potential energy that dominates over the kinetic energy in this limit. In our simulations, we see this as the effect of "freezing" of the interface position at large enough $p$. [By its nature, a rough interface experiences local fluctuations that gradually lead to its global drift.] The zero-point roughening fluctuations in the smooth phase are due to the specific solitons illustrated in Fig. 4. These solitons shift the position of the interface in the $x$ direction by one step. It is also seen that they carry a topological charge associated with shifting, by one lattice period, the checkerboard density wave along the interface ( $y$ direction). These quasiparticles also carry a particle charge $\pm 1 / 2$, since a single-particle hopping event translates the soliton by two lattice spacings, see Fig. 4. In

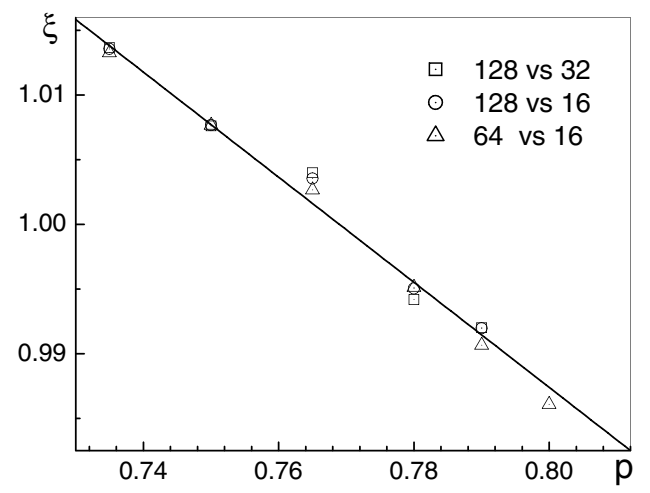

FIG. 3. Parameter $\xi$ for different data sets as a function of $p$. The solid line is a linear fit. The errors are of the order of the symbol size. 
(a)

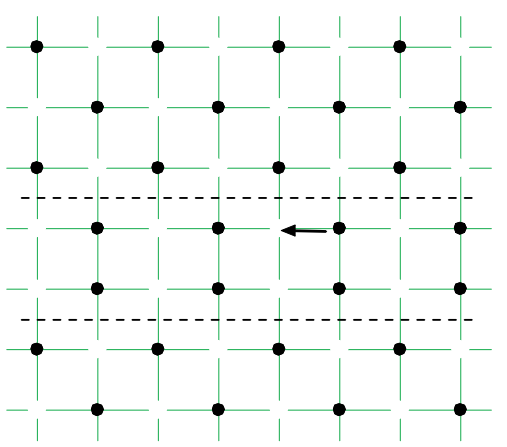

(b)

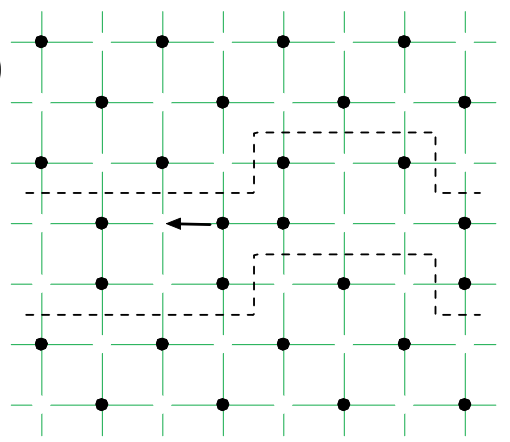

(c)

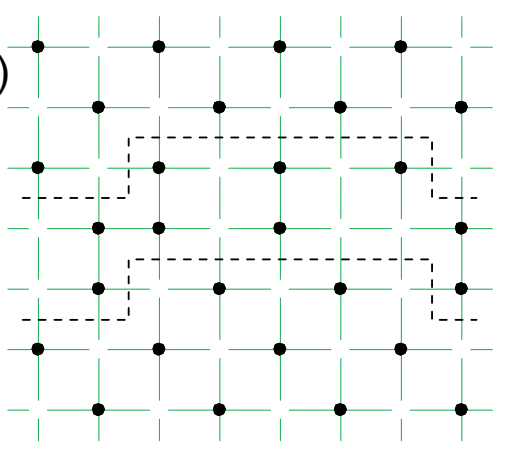

FIG. 4 (color online). A sketch of the wall roughening mechanism due to spinons. (a) a "frozen" wall (the arrow shows a singleparticle hopping event, which generates the configuration (b), and the dashed lines are to facilitate the wall gazing); (b) a configuration, featuring a pair of spinons; (c) with a single-particle hopping over one lattice site the spinon is shifted by two lattice periods, which means that the spinon particle charge is $1 / 2$.

view of their fractional particle charge it is conventional to call these solitons spinons [16]. It turns out that in the insulating phase spinons are the lowest particle-charge carrying elementary excitations. - The snapshots of the world line configurations cross sections in planes perpendicular to the $\tau$ direction reveal structures identical to those of Fig. 4. It is reasonable to assume then that the transition from the insulating to superfluid phase is due to the proliferation of spinons, in analogy to the superfluid transition in a standard 1D checkerboard solid, which is also driven by spinons. Hence, we arrive at a picture where both superfluid and roughening transitions occur simultaneously, being driven by proliferation (condensation) of one and the same quasiparticle mode.

In conclusion, we have demonstrated that an interface layer in a normal solid may exhibit superfluidity in a wide range of parameters. This result may be of direct relevance to NCMI of solid ${ }^{4} \mathrm{He}$ discovered by Kim and Chan [1], supporting the interpretation in terms of the superfluidity of microcrystallite interfaces. We have studied numerically superfluid-insulator quantum phase transitions in particlehole symmetric interfaces in 2D and 3D models of the lattice checkerboard solid. In 3D, the transition is in the $U(1)$ universality class implying that the interface is smooth. In 2D, where the interface is a 1D Luttinger liquid, we observe a Kosterlitz-Thouless type transition at the Luttinger-liquid parameter $g=1 / 2$, which implies that the interface is rough on the superfluid side. We argue that the 1D interface becomes smooth simultaneously with becoming insulating, since the onset of superfluidity and roughening are due to proliferation of the same quasiparticles which (i) have particle charge $\pm 1 / 2$, (ii) represent defects in the checkerboard order, and (iii) are kinks shifting the interface in the perpendicular direction by one lattice spacing.

We are grateful to Subir Sachdev for a discussion of the results. The research was supported by the National Science Foundation under Grant Nos. PHY-0426881 and PHY-0426814, by NASA under Grant No. NAG32870, and by PSC CUNY Grant No. 665560035.
[1] M. W. Kim and M. H. W. Chan, Nature (London) 427, 225 (2004); Science 305, 1941 (2004).

[2] N. V. Prokof'ev and B. V. Svistunov, cond-mat/0409472.

[3] M. W. Meisel, Physica B (Amsterdam) 178, 121 (1992).

[4] J.R. Beamish, Proceedings of the International Symposium on Quantum Fluids and Solids, Trento, 2004 (to be published).

[5] D. M. Ceperley and B. Bernu, Phys. Rev. Lett. 93, 155303 (2004).

[6] A. F. Andreev and I. M. Lifshitz, Zh. Eksp. Teor. Fiz. 69, 1107 (1969); G. V. Chester, Phys. Rev. A 2, 256 (1970).

[7] G. Schmid, S. Todo, M. Troyer, and A. Dorneich, Phys. Rev. Lett. 88, 167208 (2002).

[8] G. G. Batrouni and R. T. Scalettar, Phys. Rev. Lett. 84, 1599 (2000); F. Hébert et al., Phys. Rev. B 65, 014513 (2002).

[9] L. N. Bulaevski and V. L. Ginzburg, Sov. Phys. JETP 18, 530 (1964).

[10] M. Wallin, E. S. Sørensen, S. M. Girvin, and A. P. Young, Phys. Rev. B 49, 12115 (1994).

[11] N. Prokof'ev and B. Svistunov, Phys. Rev. Lett. 87, 160601 (2001); F. Alet and E. S. Sørensen, Phys. Rev. E 67, 015701(R) (2003); N. V. Prokof'ev and B. V. Svistunov, Phys. Rev. Lett. 92, 015703 (2004).

[12] E. L. Pollock and D. M. Ceperley, Phys. Rev. B 36, 8343 (1987).

[13] If the interfaces in ${ }^{4} \mathrm{He}$ polycrystal prove superfluid, the scenario for their solidification may be different because of the absence of the commensurability. It is likely that in this case the transition will generically be in the universality class of a disordered system, with the Bose glass type of the insulating phase.

[14] M. P. A. Fisher, P. B. Weichman, G. Grinstein, and D. S. Fisher, Phys. Rev. B 40, 546 (1989).

[15] S. Coleman, Phys. Rev. D 11, 2088 (1975); S. T. Chui and P. A. Lee, Phys. Rev. Lett. 35, 315 (1975); F. D. M. Haldane, Phys. Rev. Lett. 47, 1840 (1981).

[16] J. des Cloizeaux and J. Pearson, Phys. Rev. 128, 2131 (1962); L. D. Faddeev and L. A. Takhatajan, Russ. Math. Surv., 34, 11 (1979); Phys. Lett. A 85, 375 (1981). 